NOTE: this document is a pre-press version of this article, published in 2018 by:

Bioinformatics, bty1039,https://doi.org/10.1093/bioinformatics/bty1039

The article on the journal website is at:

https://academic.oup.com/bioinformatics/advance-articleabstract/doi/10.1093/bioinformatics/bty1039/5267824

There may be slight formatting and editorial differences from the published version. 
Data and text mining

\title{
multiview: a software package for multiview pattern recognition methods
}

\section{Samir Kanaan-Izquierdo 1,3,4*, Andrey Ziyatdinov ${ }^{2}$, Maria Araceli Burgueño ${ }^{5}$, and Alexandre Perera-Lluna ${ }^{1,3,4}$}

\author{
${ }^{1}$ Centre de Recerca en Enginyeria Biomèdica, Universitat Politècnica de Catalunya, Pau Gargallo 5, Barcelona, 08028, Spain \\ ${ }^{2}$ Department of Epidemiology, Harvard T.H. Chan School of Public Health, Boston, Massachusetts, United States of America \\ ${ }^{3}$ CIBER of Bioengineering, Biomaterials and Nanomedicine (CIBER-BBN), Barcelona, Catalonia, Spain \\ ${ }^{4}$ Institut de Recerca Sant Joan de Deu, Esplugues de Llobregat, Spain. \\ ${ }^{5}$ Universitat Oberta de Catalunya, Barcelona, Spain. \\ *To whom correspondence should be addressed. \\ Associate Editor: $X X X X X X X$
}

Received on XXXXX; revised on XXXXX; accepted on XXXXX

\begin{abstract}
Summary: Multiview datasets are the norm in bioinformatics, often under the label multi-omics. Multiview data is gathered from several experiments, measurements or feature sets available for the same subjects. Recent studies in pattern recognition have shown the advantage of using multiview methods of clustering and dimensionality reduction; however, none of these methods are readily available to the extent of our knowledge. Multiview extensions of four well-known pattern recognition methods are proposed here. Three multiview dimensionality reduction methods: multiview t-distributed stochastic neighbour embedding, multiview multidimensional scaling, and multiview minimum curvilinearity embedding, as well as a multiview spectral clustering method. Often they produce better results than their single-view counterparts, tested here on four multiview datasets.

Availability and implementation: R package at the B2SLab site: http://b2slab.upc.edu/software-andtutorials/ and Python package: https://pypi.python.org/pypi/multiview.

Contact: samir.kanaan@upc.edu

Supplementary information: Supplementary information is available at Bioinformatics online.
\end{abstract}

\section{Introduction}

Multiview datasets comprise several data matrices or views, where each matrix contains the result of a different measurement or experiment on the same subjects. Examples of data views in the bioinformatics field are: gene sequencing and expression, metabolomic data, phenotypes, medical imaging. True multiview methods simultaneously process two or more data views to produce a single result coherent with all of them. Several studies show that true multiview methods perform better than single-view solutions (Zhang et al., 2015; Zhao et al., 2014).

Multiview methods for unsupervised tasks are specially useful, as there is no a priori knowledge on classes and consequently it is more difficult to choose the right data view. Even though several multiview methods have been proposed, to the extent of our knowledge none of them is available as open software. This paper presents multiview extensions to

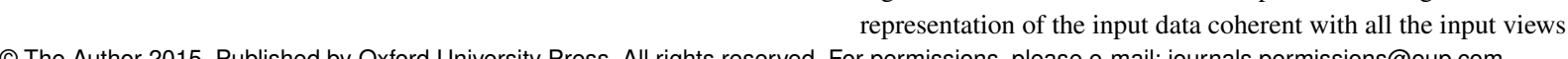

four well known pattern recognition methods: (1) t-distributed stochastic neighbour embedding ( $t$-SNE) (Van Der Maaten et al., 2008), (2) Multidimensional scaling (MDS) (Kruskal, 1964) and (3) Minimum curvilinearity embedding $M C E$ (Cannistraci et al., 2010, 2013) are standard dimensionality reduction and data visualization methods. (4) Spectral clustering (SC) (Shi and Malik, 2005) is an advanced clustering method that can identify non-convex clusters. The new multiview methods are implemented as open source $\mathrm{R}$ and Python packages. They are described here along with some application examples and results.

\section{Methods}

Multiview dimensionality reduction methods receive a set of $v \geq 2$ high-dimensional data views and produce a single, low-dimensional 


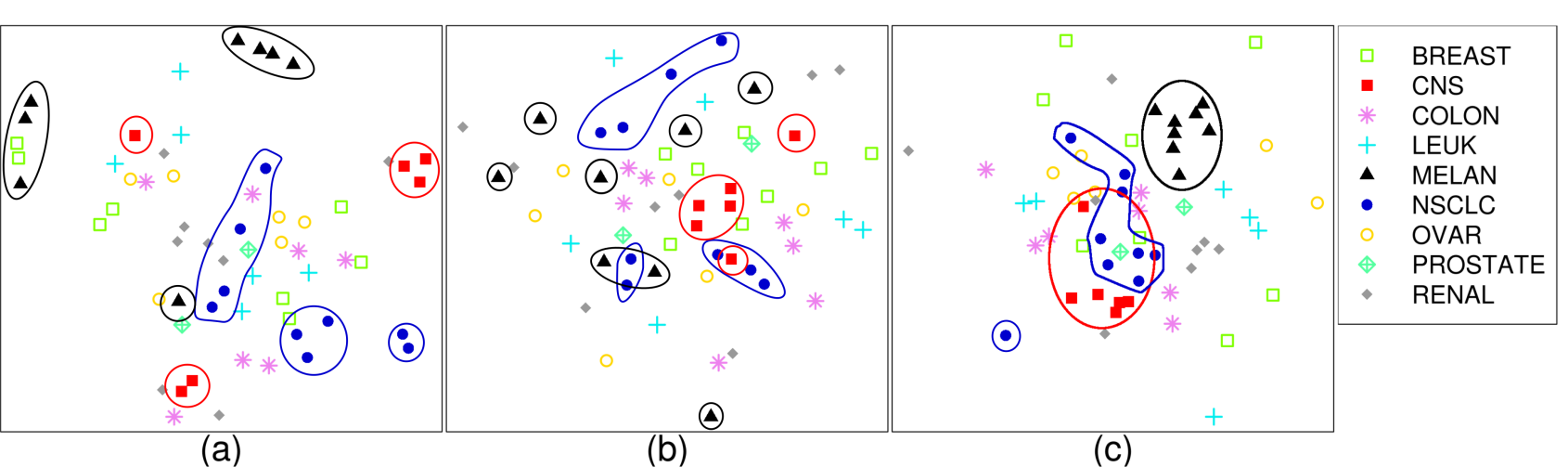

(a)

(b)

(c)

Fig. 1. Multidrug cell line data projection. (a) t-SNE on the ABC expression levels; (b) t-SNE on the reaction to drugs; (c) multiview t-SNE.

Multiview t-SNE (mv-tsne) computes a neighborhood probability matrix $P_{1 \leq i \leq v}$ for each input matrix. mv-tsne merges the $v$ probability matrices applying the expert opinion pooling results from (Abbas, 2009). More specifically, it obtains a single probability matrix using the log-linear pooling $P=r \prod_{i=1}^{v} P_{i}^{\omega_{i}}$, where $r$ is a normalization factor and the optimal $\omega_{i}$ exponents are determined in an optimization stage. Afterwards, the t-SNE optimization stage is applied to $P$ to find the optimal data projection

Multiview MDS (mv-mds) double-centers the input matrices and computes the first $k$ common eigenvectors using a variation of the CPCA method proposed in (Trendafilov, 2010). The result is the orthogonal matrix $W$ such that the preprocessed input matrices can all be expressed as $L_{i}^{\prime}=W^{T} L_{i} W, i=1,2, \ldots, v$. Hence, the common lowdimensional projection of the original multiview data are the first $k$ common eigenvectors computed by CPCA, where $k$ is the desired dimensionality of the projection.

Multiview MCE (mv-mce) is a multiview extension to MCE. Origina MCE computes a distance matrix as the shortest paths between all data points over their minimum spanning tree, then applies MDS to produce a low-dimensional representation of the data. mv-mce computes the shortest paths over the minimum spanning tree over each of the input views, then applies mv-mds to produce a single low-dimensional representation of the data.

Given a multiview dataset, with $v \geq 2$ data views, multiview clustering methods find a clustering assignment that is expected to be coherent with the $v$ input data views.

Multiview spectral clustering (mv-sc) (Kanaan-Izquierdo et al., 2018) computes the clustering of a multiview dataset in three steps: first it computes the Laplacian matrices of all input views; second it computes the first $k$ common eigenvectors of the data using CPCA (Trendafilov, 2010); finally it computes the clustering assignment using K-means. CPCA guarantees a decreasing sum of the eigenvalues associated to each eigenvector, thus conserving the eigengaps: $\delta^{(i)}=\sum_{c=1}^{C} \lambda_{c}^{(i)}-$ $\sum_{c=1}^{C} \lambda_{c}^{(i+1)} \geq 0 \forall i=1,2, \ldots k$. This satisfies the matrix perturbation theory condition and consequently mv-sc produces a stable subspace on which the data clustering can be obtained.

\section{Results}

Package multiview has been tested on four multiview datasets: multidrug cell line dataset (Szakács et al., 2004), the Berkeley protein dataset (Lanckriet et al., 2004), CORA dataset (McCallum and Nigam, 1998), and a dataset of features from 2D electrophoresis images of cerebrospinal fluid, in the context of a study on neuropathies (Pattini et al., 2008) (2DE-CSF).
Table 1. Clustering quality on the datasets used.

\begin{tabular}{rrrrr}
\hline & & Best & Stacked & mv-sc \\
\hline Multidrug cell & Purity & 0.469 & 0.469 & $\mathbf{0 . 5 4 2}$ \\
& NMI & 0.483 & 0.483 & $\mathbf{0 . 5 5 0}$ \\
\hline Berkeley protein & Purity & 0.785 & 0.796 & $\mathbf{0 . 8 0 7}$ \\
& NMI & 0.309 & 0.295 & $\mathbf{0 . 3 4 6}$ \\
\hline CORA & Purity & 0.335 & 0.350 & $\mathbf{0 . 3 8 4}$ \\
& NMI & 0.135 & 0.186 & $\mathbf{0 . 1 8 9}$ \\
\hline
\end{tabular}

mv-tsne has been applied to the multidrug cell line dataset. Figure shows the results, where subplots (a) and (b) correspond to standard tSNE applied to each view, and subplot (c) corresponds to the multiview projection produced by mv-tsne. mv-tsne finds the common traits of severa cell locations (notably MELAN, CNS and NSCLC), even if those cell groups appear scattered on the single view projections (a) and (b). mv-tsne an mv-mds projections are also quantitatively better than those produced by the single view equivalent methods.

Table 1 shows the clustering purity and normalized mutual information on the tested datasets using single views, stacked data and mv-sc

Finally, mv-mce has been applied to the 2DE-CSF dataset in order to obtain a $2 \mathrm{D}$ representation of the 2,050 features in the dataset. These features have been split in two blocks according to an initial clustering (900 and 1150 features), which in turn have been used as input data views for mv-mce. Figure 2 shows the resulting projection and its connection with the four subject classes in the study.

\section{Conclusions}

Package multiview provides multiview extensions of widely used pattern recognition methods that yield higher quality results than their single view counterparts. The dimensionality reduction methods may help to discover underlying patterns in the data that may not be apparent when working with a data view alone. Moreover they provide a single view representation of multiview data, allowing their use with classical methods. The multiview spectral clustering method produces better clustering assignments than single-view spectral clustering. Besides, all the methods presented can process any number and type of input data views. In conclusion, package multiview, available in $\mathrm{R}$ and Python, provides potentially useful and widely applicable pattern recognition methods to the bioinformatics community, so this package makes a relevant contribution. 


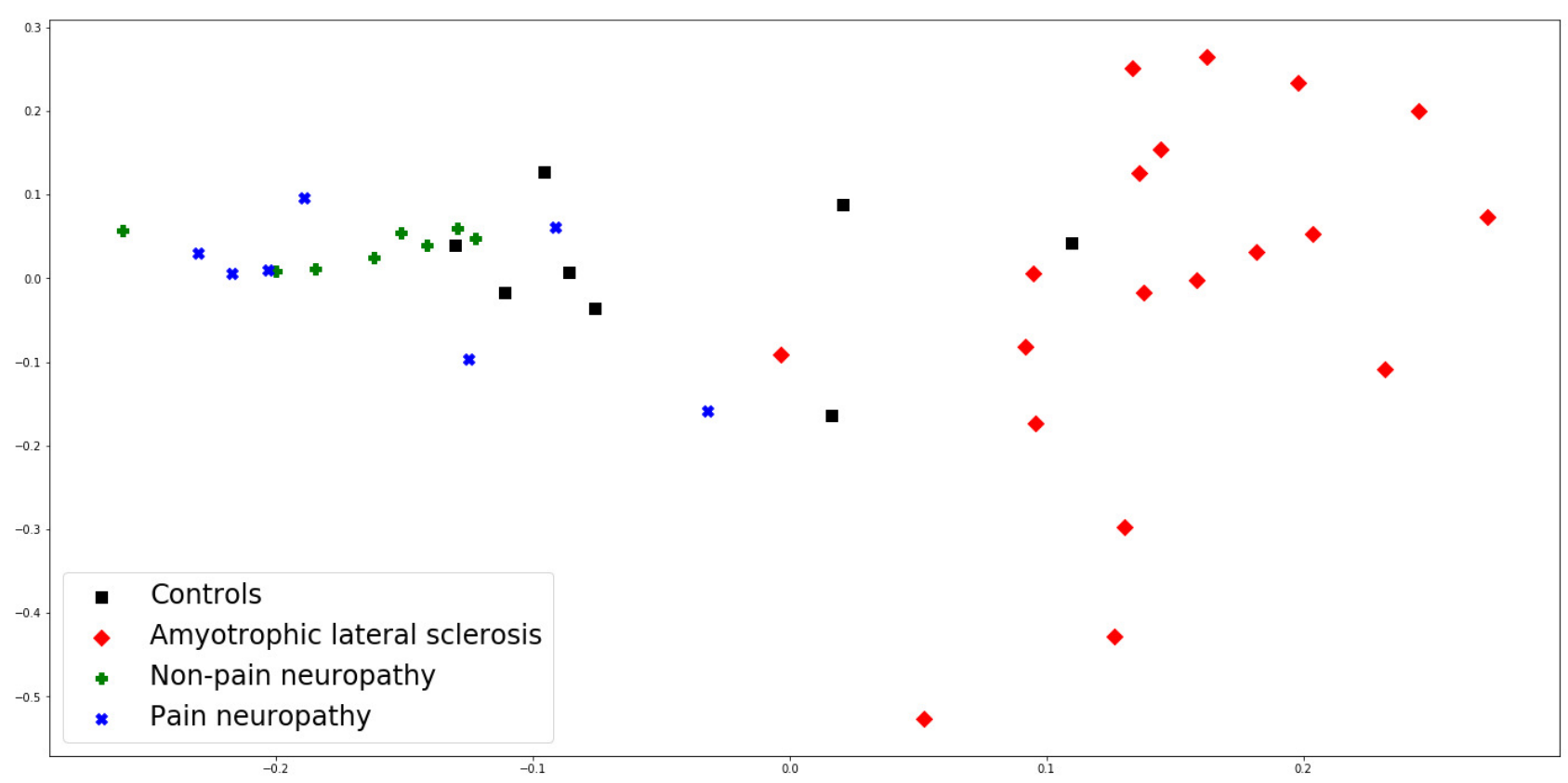

Fig. 2. Multiview minimum curvilinearity embedding projection of the 2DE-CSF dataset.

\section{Funding}

This work was supported by TEC2013-44666-R, TEC2014-60337-R and the 2009SGR-1395 consolidated research group of the Generalitat de Catalunya, Spain. CIBER-BBN is an initiative of the Spanish ISCIII.

\section{References}

Abbas, A. E. (2009). A Kullback-Leibler View of Linear and Log-Linear Pools. Decision Analysis, 6(1), 25-37.

Cannistraci, C. V., Ravasi, T., Montevecchi, F. M., Ideker, T., and Alessio, M. (2010). Nonlinear dimension reduction and clustering by minimum curvilinearity unfold neuropathic pain and tissue embryological classes. Bioinformatics, 26(18), i531-i539.

Cannistraci, C. V., Alanis-Lobato, G., and Ravasi, T. (2013). Minimum curvilinearity to enhance topological prediction of protein interactions by network embedding. Bioinformatics, 29(13), i199-i209.

Kanaan-Izquierdo, S., Ziyatdinov, A., and Perera-Lluna, A. (2018). Multiview and multifeature spectral clustering using common eigenvectors. Pattern Recognition Letters, 102, 30 - 36 .

Kruskal, J. B. (1964). Multidimensional scaling by optimizing goodness of fit to a nonmetric hypothesis. Psychometrika, 29(1), 1-27.

Lanckriet, G. R. G., De Bie, T. Cristianini, N., Jordan, M. I., and Noble, W. S. (2004). A statistical framework for genomic data fusion. Bioinformatics (Oxford,
England), 20(16), 2626-35.

McCallum, A. and Nigam, K. (1998). A Comparison of Event Models for Naive Bayes Text Classification. AAAI/ICML-98 Workshop on Learning for Text Categorization, pages 41-48.

Pattini, L., Mazzara, S., Conti, A., Iannaccone, S., Cerutti, S., and Alessio, M. (2008). An integrated strategy in two-dimensional electrophoresis analysis able to identify discriminants between different clinical conditions. Experimental Biology and Medicine, 233(4), 483-491.

Shi, J. and Malik, J. (2005). Normalized Cuts and Image Segmentation Normalized Cuts and Image Segmentation. Pattern Analysis and Machine Intelligence, IEEE Transactions on, 22(March), 888-905.

Szakács, G., Annereau, J.-P., Lababidi, S., Shankavaram, U., Arciello, A., Bussey, K. J., Reinhold, W., Guo, Y., Kruh, G. D., Reimers, M., Weinstein, J. N., and Gottesman, M. M. (2004). Predicting drug sensitivity and resistance: Profiling $\mathrm{ABC}$ transporter genes in cancer cells. Cancer Cell, 6(2), 129-137.

Trendafilov, N. T. (2010). Stepwise estimation of common principal components. Computational Statistics and Data Analysis, 54(12), 3446-3457.

Van Der Maaten, L., Hinton, G., and van der Maaten, G. H. (2008). Visualizing Data using t-SNE.

Zhang, L., Zhang, Q., Zhang, L., Tao, D., Huang, X., and Du, B. (2015) Ensemble manifold regularized sparse low-rank approximation for multiview feature embedding. Pattern Recognition, 48(10), 3102-3112.

Zhao, X., Evans, N., and Dugelay, J.-L. (2014). A subspace co-training framework for multi-view clustering. Pattern Recognition Letters, 41(0), 73-82. 